\title{
A New Observational Upper Limit to the Low Redshift Ionizing Background Radiation
}

\author{
Ray J. Weymann \\ Carnegie Observatories, 813 Santa Barbara Street, Pasadena CA 91101 \\ rjw@ociw.edu \\ Stuart N. Vogel \\ Department of Astronomy, University of Maryland, College Park, MD 20742 \\ vogel@astro.umd.edu \\ Sylvain Veilleux \\ Department of Astronomy, University of Maryland, College Park, MD 20742 \\ veilleux@astro.umd.edu \\ Harland W. Epps \\ Lick Observatory, University of California Santa Cruz, Santa Cruz, CA 95064 \\ epps@ucolick.org
}

\begin{abstract}
We report a new Fabry-Perot search for $\mathrm{H} \alpha$ emission from the intergalactic cloud HI 1225+01 in an attempt to measure the low redshift ionizing background radiation. We set a new $2 \sigma$ upper limit on $\mathrm{H} \alpha$ emission of $8 \mathrm{mR}\left(5 \times 10^{-20}\right.$ ergs $\left.\mathrm{cm}^{-2} \mathrm{~s}^{-1} \operatorname{arcsec}{ }^{-2}\right)$. Conversion of this limit to limits on the strength of
\end{abstract}


the ionizing background requires knowledge of the ratio of the projected to total surface area of this cloud, which is uncertain. We discuss the plausible range of this ratio, and within this range find that the strength of the ionizing backround is in the lower range of, but consistent with, previous observational and theoretical estimates.

Subject headings: Cosmology: diffuse radiation, Galaxies: intergalactic medium

\section{INTRODUCTION}

The value of the local ionizing flux is of interest for several reasons: 1) It plays an important role in setting the level of ionization of the low density hydrogen gas which gives rise to the Ly $\alpha$ forest. Recent simulations have been remarkably successful in reproducing the main features of the Ly $\alpha$ forest from high redshifts to zero redshift (Davé \& Tripp 2001). As discussed in detail in $\S 4$, for a given set of cosmological parameters, these simulations are now able to make predictions about the value of the local $\mathrm{H}$ I photoionization rate, and it would be valuable to determine empirically the value of this rate to compare it with the simulations. With a sufficiently accurate determination of its value and further forthcoming data on the low redshift Ly $\alpha$ forest, together with further improvements in the simulations it may be possible to set some constraints on these cosmological parameters, especially the baryon number. 2) A limit on the photoionization rate in locations which are free of local ionizing sources (i.e. star-forming regions or ionization due to high energy particles in the neighborhood of galaxies) can then be used to assess the relative importance of such local sources near a galaxy compared to the non-local, global value. In particular, there is currently some controversy concerning the ionization source for some high velocity

clouds in our own galaxy (Weiner, Vogel, \& Williams 2000; Bland-Hawthorn \& Putnam 2001). 3) Estimates of the photoionization rate due to the cumulative effect of active galactic 
nuclei can now be carried out with fair confidence (Shull et al. 1999, hereafter SRGPF); but there remain significant uncertainties associated primarily with the luminosity functions of these objects and with the evaluation of the opacity associated with intervening neutral hydrogen. 4) Similar remarks apply to the contribution from escaping radiation shortward of the Lyman limit from hot stars in galaxies. However, this estimate is also dependent upon the fraction of the Lyman continuum radiation assumed to escape from the galaxies, and direct observational evidence about the magnitude of this escape fraction is still somewhat scanty.

For these reasons, we have previously set limits on the value of the non-local ionization rate at zero redshift by setting limits on the $\mathrm{H} \alpha$ flux from an isolated, large, $\mathrm{H}$ I cloud which is optically thick to the Lyman continuum (Vogel et al. 1995, hereafter VWRH). The essence of the method is very simple. Illumination by ionizing radiation of an $\mathrm{H} I$ cloud that is optically thick to the Lyman continuum results in $\mathrm{H} \alpha$ emission that is well described by Case $\mathrm{B}$ recombination theory. Accordingly, approximately one $\mathrm{H} \alpha$ photon escapes for every two incident Lyman continuum photons; the exact value depends only mildly on parameters that are reasonably well known. The one significant uncertainty is the ratio of the observed projected surface area to that illuminated by the ionizing radiation; however, the uncertainty is perhaps a factor of two, and can be better determined for objects where the geometry is known, such as galactic disks. The expected $\mathrm{H} \alpha$ radiation is extremely faint - based on predictions for the low redshift metagalactic ionizing flux (e.g., SRGPF), the $\mathrm{H} \alpha$ surface brightness is expected to be only a few milliRayleighs (for $\mathrm{H} \alpha, 1 \mathrm{mR}=$ $\left.5.67 \times 10^{-21} \mathrm{ergs} \mathrm{cm}^{-2} \mathrm{~s}^{-1} \operatorname{arcsec}^{-2}\right)$. By comparison, the observed $\mathrm{H} \alpha$ emission from objects ionized by other sources is significantly brighter: high velocity clouds known to be within a few kpc are typically 60-200 mR (Tufte, Reynolds, \& Haffner 1998); most compact high velocity clouds lie between 30 to $300 \mathrm{mR}$ (Weiner, Vogel, \& Williams 2000); the Magellanic Stream ranges up to more than $1000 \mathrm{mR}$ (Weiner \& Williams 1996); a brightness of $90 \mathrm{mR}$ 
was measured in the outer disk of NGC 253 (Bland-Hawthorn, Freeman, \& Quinn 1997); and the diffuse extraplanar ionized gas imaged in edge-on spirals is typically brighter than 1000 mR (Rand, Kulkarni, \& Hester 1990; Veilleux, Cecil, \& Bland-Hawthorn 1995). As a result of its expected faintness, detection of $\mathrm{H} \alpha$ emission due to ionization by the metagalactic ionization requires observation of extended clouds using specialized techniques.

There exist other approaches to an empirical value for the global zero redshift ionization rate. However, several of these methods suffer from significant limitations. At higher redshifts, the proximity effect (Bajtlik, Duncan, \& Ostriker 1988; Bechtold 1994) has been used to estimate the value of the metagalactic ionizing flux; however at low redshift the sparseness of higher resolution data and the relative sparseness of the Ly $\alpha$ forest itself makes this method currently less useful. In addition, there are some issues about the interpretation of the proximity effect (e.g., the clustering properties of matter in the vicinity of QSOs; see Scott et al. (2000) for further discussion).

A potentially very powerful technique involves observing the rapid transition from a mostly neutral hydrogen disk to a mostly ionized disk as one moves outward along the radius of spiral galaxies. From the value of this transition column density, one can infer the flux of the background ionizing radiation given a model for the vertical distribution of gas. The basic idea for this technique goes back to papers by Sunyaev (1969) and Felten \& Bergeron (1969). The historical development of this idea is summarized by Maloney (1993) who applied it to realistic galaxy models. Maloney gives a careful treatment of the radiative transfer equation and discusses a wide range of galaxy parameters. His results show that the derived ionizing flux is relatively insensitive to the dynamical models adopted for the galaxy. The application to NGC3198, which has the most sensitive HI observations in this context, and whose geometry and dynamics are not very uncertain, yielded a transition column density of about $5 \times 10^{19} \mathrm{~cm}^{-2}$ and from this Maloney deduced that the flux of 
ionizing photons is in the range $5 \times 10^{3}$ to $5 \times 10^{4}$ photons $\mathrm{cm}^{-2} \mathrm{sec}^{-1}$. Shortly after this work Dove \& Shull (1994) published a similar analysis and confirmed these results. They also showed that the results are not very sensitive to the assumed spectral index of the ionizing background.

Application of this method to other objects has also been carried out. In particular, as noted above, measurements of $\mathrm{H} \alpha$ emission beyond the $\mathrm{HI}$ truncation radius were obtained by Bland-Hawthorn, Freeman, \& Quinn (1997) in NGC253. In this case, however, these authors noted that the inferred surface brightness was higher than could likely arise only from the metagalactic radiation field. In addition, the ratio of [NII] $\lambda 6548$ to $\mathrm{H} \alpha$ emission was unusually high. These authors favored a model in which a warp in the outer disk allowed ionizating flux from the inner young star population to dominate the ionization. In NGC253 the ratio of the HI disk radius to the optical disk radius is considerably smaller than in NGC3198. Nevertheless, Dove \& Shull (1994) do not reject the possibility that hidden local sources of ionization may contribute significantly to the ionizing flux even in NGC3198.

We note that there appear to be instances where no abrupt truncation of $\mathrm{H}$ I occurs to column densities which are smaller than $\sim 5 \times 10^{19} \mathrm{~cm}^{-2}$. For example, Walsh, StaveleySmith \& Oosterloo (1997) present HI images of NGC 289, a giant gas-rich low-surfacebrightness spiral, and show HI contours down to a column density of $1 \times 10^{19} \mathrm{~cm}^{-2}$; they note that no truncation is evident. In DDO 154, Carignan \& Purton (1998) find no truncation, down to a level of $1 \times 10^{19} \mathrm{~cm}^{-2}$. This might be indicative of a value for the ionizing background somewhat lower than that derived for NGC3198, but it is also possible that the low observed HI column densities are a consequence of beam dilution; with better angular resolution, the actual column densities might be observed to be substantially higher. In addition, however, as emphasized by Maloney (2001, private communication), the abruptness of the $\mathrm{H}$ I cutoff depends upon the run of the total hydrogen column density which is not $a$ 
priori known, and the ratio of the neutral to total hydrogen column density is sensitive to the total hydrogen column density in the regime where the predominate ionization state switches from neutral to ionized (cf Figures 4 and 8 of Maloney 1993). In fact, the estimate of the background radiation in NGC3198 was carried out by using the observed H I column density to model the total hydrogen column density for assumed values of the ionizing background. It is the uncertainty of the true radial variation of the total column density of hydrogen at these large radii that appears to be the principal uncertainty in the determination of the background radiation using this technique.

In any event, if the relatively large value of the metagalactic ionizing flux implied by the HI truncation of NGC 3198 is correct, then the $\mathrm{H} \alpha$ emission produced by this ionization should be detectable with achievable sensitivities in instances where the HI column density is high enough to ensure that most of the ionizing photons are absorbed.

For these reasons, we continue to believe that the use of $\mathrm{H} \alpha$ surface brightness measurements as described in VWRH still provides the route to the least model-dependent estimate for the metagalactic ionizing flux, provided that (i) The targets are relatively isolated from star forming regions and (ii) that the geometry is well understood, for the reason discussed in section 3.3

Consequently, when subsequent further improvements in our instrumentation were made, we again carried out basically the same series of observations as described in VWRH, which resulted in substantial further lowering of an upper limit on the zero redshift photoionization rate. The purpose of the present paper is to describe and discuss these observations.

The selected target, HI $1225+01$, is the same as in VWRH. This large and isolated cloud of H I was discovered serendipitously by Giovanelli \& Haynes (1989), and subsequent mapping by Giovanelli, Williams, \& Haynes (1991) showed it to consist of two distinct components - a northeast component (NE) and a southwest component (SW), with a weaker 
bridge of H I connecting the two. The NE component has embedded in it stars and a small region of star formation (Turner \& MacFadyen 1997, and references therein). However the SW component shows no trace of a stellar component (Salzer et al. 1991, and references therein). For this reason, we chose to observe only the SW component since the relatively bright $\mathrm{H} \alpha$ emission from the hot stars in the NE component would mask the weak diffuse signal we are attempting to detect. Subsequently, a higher resolution H I study (40") using the VLA was carried out by Chengalur, Giovanelli \& Haynes (1995); they showed that the SW component appears to be a flattened inclined disk with a systematic velocity $V_{\text {hel }}=1260$

$\mathrm{km} \mathrm{s}^{-1}$ and a maximum rotational velocity of $\sim 13 \mathrm{~km} \mathrm{~s}^{-1}$. We discuss the properties of the SW component further in $§ 3.3 .2$. The cocoon of H I in which the optical central part of the NE component resides makes it unlikely that ionizing radiation from the hot stars in this component will ionize a significant portion of the SW component, and the isolation of HI 1225+01 from other galaxies makes the SW component an attractive target for this program.

The plan of the remainder of the paper is as follows: In $\S 2$ we describe the instrumentation and observational procedures used for the results described below and in $\S 3$ we describe the reduction procedures. We present the results in $\S 4$ along with a discussion of their implications for some of the issues described above which motivated these observations.

\section{INSTRUMENTATION AND OBSERVATIONS}

\subsection{Instrumentation}

\subsubsection{Reducing Camera}

The observations described here were carried out at the Carnegie Observatories on Las Campanas, using the duPont $2.5 \mathrm{~m}$ telescope. A reducing camera was constructed for this purpose. The optical design was carried out by H. Epps, with mechanical design by A. 
Schier and associates. The design consisted of a collimator with an on-axis beam size of 70 $\mathrm{mm}$ and an effective focal length of $525 \mathrm{~mm}$ to match the $\mathrm{f} / 7.5$ incoming beam from the Cassegrain focus of the duPont telescope. A $70 \mathrm{~mm}$ Fabry-Perot etalon (whose properties are described in $\S 2.1 .2$ ) was placed in the collimated beam. An f/2.5 camera reimages the telescope focal plane onto a SITe 2048 x 2048 CCD with 24 micron pixels, and a scale at the CCD of approximately $0.78^{\prime \prime}$ per pixel. The CCD was operated in a mode which yielded a readout noise of about 6e per pixel. However, as explained below, we were obliged to accept a tradeoff between adequate sampling of the signal and readout noise, and thus we binned the image in $4 \times 4$ pixels. The combined collimator-camera reducing camera is achromatic over a very broad range and yields an unvignetted field of about $25^{\prime}$ in diameter.

\subsubsection{Etalon}

The University of Maryland ET-70 etalon was used for this experiment. The etalon and CS100 controller are made by Queensgate. The $70 \mathrm{~mm}$ diameter etalon has a gap of 44 $\mu \mathrm{m}$, and a free spectral range (FSR) of $48.7 \AA$ at $6590.4 \AA$, the expected center of the redshifted $\mathrm{H} \alpha$. The FWHM of the instrumental response is $1.40 \AA$, and the effective bandwidth (see $§ 3.1$ ) is $1.64 \AA$. The etalon was flushed with dry nitrogen during the observations and calibrations. The etalon can be tilted so as to direct reflections out of the field of view.

A four-cavity, 6-inch square, order-isolating filter was located near the telescope focal plane. The filter has an intrinsic FWHM of $38 \AA$, with a transmission greater than $75 \%$ for

$6581 \AA<\lambda<6605 \AA$. Since the etalon FSR is $48.7 \AA$, in this wavelength range photometric calibration on continuum objects does not suffer from contamination from other orders. 


\subsection{Observations}

We observed HI 1225+01 the nights of 1997 April 7-10 using the reducing camera and Fabry-Perot etalon described in $§ 2.1$. The first three nights were photometric, while the atmospheric transparency varied during the course of the last night. Consequently, only data from the first three nights were used in the analysis described here.

To reach the very low surface brightness required for this experiment demanded careful attention to observing and reduction strategy. Both shot-noise and systematic errors could contribute to the error budget. A key strategy for minimizing systematic errors is to switch as rapidly as possible between the target and sky positions. The lower limit on the switching time is set by the need to minimize the relative error contributed by CCD read noise. As discussed in $\S 2.1$, the reducing camera was designed for this experiment with high throughput, high speed, and a large field of view. Nonetheless, due to the narrow effective bandwidth $(1.64 \AA)$, further steps were required to make the relative contribution of CCD read-noise to the total error sufficiently small. First, as noted above we used $4 \times 4$ on-chip binning of the CCD; this yielded 3.1" pixels. Hereafter we will use "pixel" to refer to one of the $4 \times 4$ binned pixels. Second, all exposures times were 900 seconds in length.

To minimize errors introduced by variations in atmospheric and instrumental response, we adopted the following observing procedure. First, we observed blank sky at a position

offset in RA to the west (referred to as W) of the subsequent HI 1225+01 target observation (referred to as GH1), with the RA offset chosen so that each pair of W and GH1 exposures was observed through very similar azimuth and elevation ranges. In this way, we sought to minimize the effects of systematic errors due to azimuthal and elevation variations in the atmospheric, telescope, and instrument response. To eliminate linear temporal drifts, we repeated an exposure on HI 1225+01 (GH2) followed by an exposure on a sky position offset to the East (E). A W-GH1-GH2-E set of four exposures, observed in the manner described, 
greatly reduced errors due to directional and temporal variations in the instrumental and atmospheric response.

We also spatially dithered each exposure by an amount varying from 5 to $45^{\prime \prime}$ in order to minimize errors due to stars and other features.

The monochromatic response in the focal plane is along a ring whose radius $r$ varies according to the usual

$$
\lambda=\lambda_{0}\left(1+(r / f)^{2}\right)^{-1 / 2}
$$

where $\lambda_{0}$ is the wavelength that transmits on-axis and $f$ is the camera focal length. As usual, $\lambda_{0}$ (and hence $\lambda$ ) is adjusted by varying the etalon gap spacing, $l$, according to $n \lambda=2 \mu l \cos \theta$, where $n$ is the order, $\mu$ is the refractive index of the nitrogen gas in the etalon gap, and $\theta$ is the angle with respect to the optical axis.

In Figure 1 we show a typical 900 second exposure toward the target position. Due to the large field, non-zero etalon tilt angle, and the quadratic nature of the wavelength response (e.g., equation 1), three to four orders are present; i.e. three to four rings are present for each night sky line. The on-axis order is 136, with orders 135, 134, and 133 also present off the optical axis. With our large, binned pixels, the quadratic nature of the wavelength response results in a wavelength shift comparable to the spectral resolution across a pixel for orders 135,134 , and 133. Since the wavelength resolution is consequently slightly degraded for the outer orders, we extract separate spectra for each order.

By the well known Jacquinot relation, $\Omega R=2 \pi$ (where $\Omega$ is the angular area and $R$ is the resolution), the angle on the sky subtended by a complete monochromatic ring is independent of radius $r$. Clearly, we want as much as possible of the ring corresponding to the redshifted $\mathrm{H} \alpha$ line to be projected within the HI $1225+01$ cloud. But we also want as 
Fig. 1.- Flat-fielded 900-second exposure toward target position. The image has been clipped where the flat-field response drops below 60\%. Most of the rings are produced by $\mathrm{OH}$ night sky emission; the brighter lines are labeled. Note that the same lines appear in each order. The brightest line (OH $6577 \AA$ ) is clipped in some places because the flat-field response near the edge of the filter passband drops below 60\%. The Galactic [N II] line also appears. The diameter of the field of view is $\sim 25^{\prime}$.

much spectral coverage on both sides of $\mathrm{H} \alpha$ as possible. Coverage at shorter wavelengths extends to the blocking filter cutoff at $6577 \AA$ and includes the Galactic [NII] $6584 \AA$ line and the atmospheric $\mathrm{OH} 6577 \AA$ line. However, to observe emission at a given $\lambda$ (in particular around 6590.4 $\AA$, the wavelength expected for the H $\alpha$ centroid in HI 1225+01 SW; see §3.2), equation (1) shows that the maximum wavelength $\lambda_{0}$ (i.e. the wavelength observed on axis) increases as $r$ is increased. In order to keep a substantial fraction of the $6590.4 \AA$ ring within the cloud boundary, we used a ring radius of 55-65 pixels (170-200"). This keeps roughly half of the ring within the cloud boundary but cuts off the red end of this spectral order at approximately $6593 \AA$ which yields a rather short range of spectrum to the red of the redshifted $\mathrm{H} \alpha$ over which to define the continuum.

The etalon and the blocking filter are normally tilted to direct reflections out of the field of view. To maximize the ring area on target, we chose an intermediate tilt angle for the etalon such that the entire ring at $6590.4 \AA$ lies within the field of view.

As shown by equation (1), the wavelength transmitted by the etalon varies as a function of position. The usual method for constructing a spectrum is to obtain multiple exposures, stepping the etalon gap $l$ through a full free spectral range, or at least sufficiently to fully sample the line profile and continuum at all positions. However, the long exposure times make this impractical. Instead, we know that $\mathrm{H} \alpha$ emission ionized by metagalactic radiation will be nearly uniform in brightness for regions that are optically thick to the Lyman continuum 
(as described in more detail in §3.3). Consequently, we can assemble the spectrum using the varying spectral information provided by the different pixels in the field of view. It is essential that the pixels used for the $\mathrm{H} \alpha$ portion of the spectrum be limited to those pixels that lie towards regions of HI $1225+01$ that are optically thick to the Lyman continuum. Pixels which transmit non- $\mathrm{H} \alpha$ wavelengths are not restricted to lie towards the cloud.

Figure 2 shows the average sky spectra obtained for one night for orders 136 and 135, along with the measured transmission of the blocking filter in the tilted configuration used for the observations. Three bright $\mathrm{OH}$ atmospheric lines are evident at 6577, 6596, and $6604 \AA$, along with a weak $\mathrm{OH}$ line near $6592 \AA$; only the 6577 and 6592 lines lie within the spectral coverage of order 136. Also apparent is a bump near $6583 \AA$ corresponding to Galactic [N II]. Continuum due to light scattered from the atmosphere, zodiacal dust, and Galactic sources is also present at a level of $\sim 6 \times 10^{-18} \operatorname{ergs~cm}^{-2} \mathrm{~s}^{-1} \operatorname{arcsec}^{-2} \AA^{-1}$.

For flux calibration, we observed standard stars each night; the stars selected included EG 274, LTT 4364, LTT 6248, and LTT 7379, using equation 3 in $\S 3.1$ to obtain absolute fluxes.

Most Fabry-Perot programs acquire observations of a diffuse monochromatic calibration source at intervals of 30 to 60 minutes during the night to monitor and correct the wavelength response. Our program yields high signal/noise ratio spectra of the atmospheric night sky lines; as described in $\S 3$ these can be used for wavelength calibration. Since they are observed simultaneously with the target, using them provides a more accurate calibration and also minimizes the need for additional calibration observations during night time observing.

Daily calibrations were acquired. These included bias and dark frames, dome and twilight flats with the etalon gap set to the spacings used during the night, and neon calibration cubes. We varied the tilt angle for the etalon from night to night, and obtained calibrations appropriate to each night. 


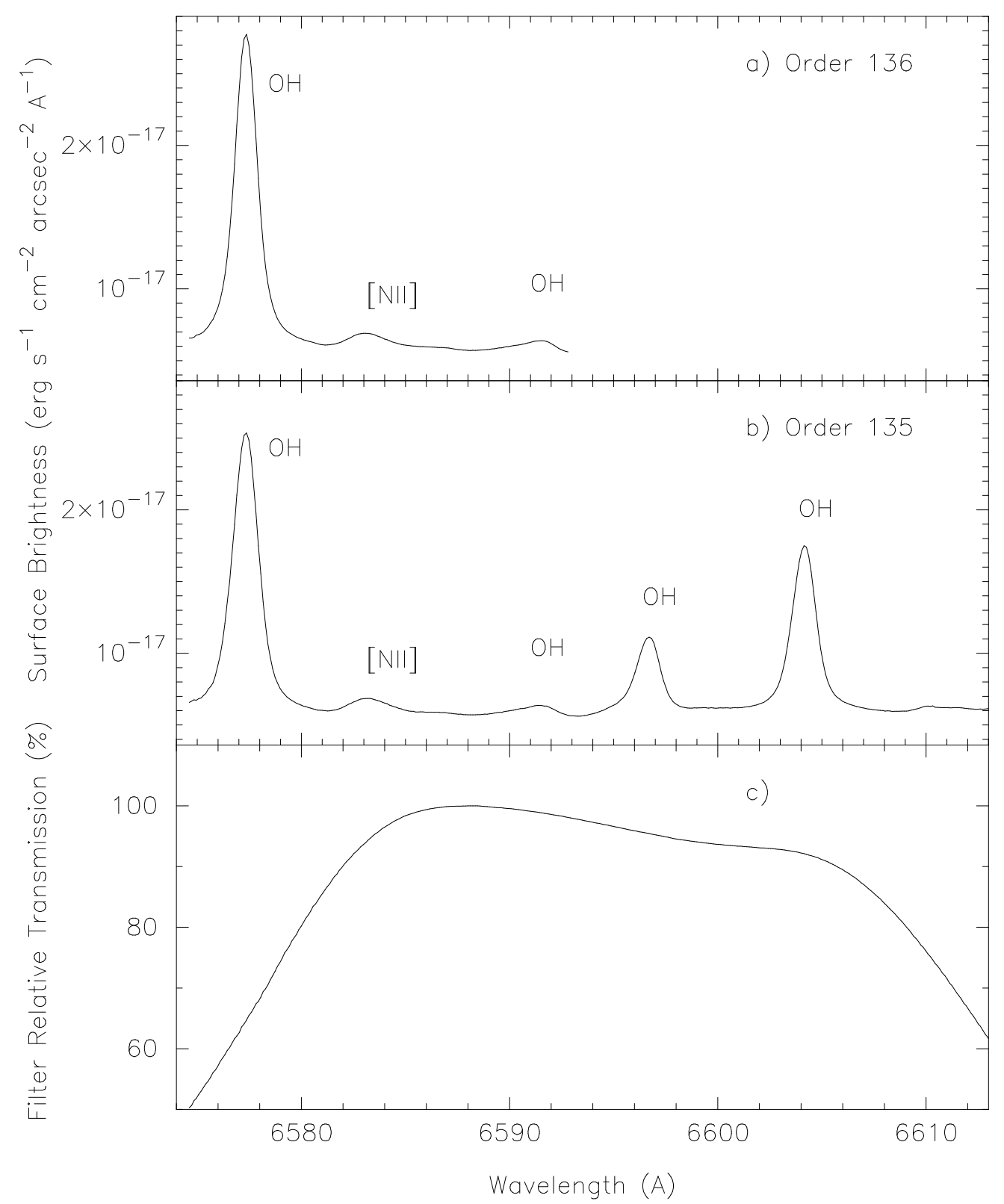

Fig. 2.- (a) Spectrum for order 136. Two night-sky OH lines are labeled, along with the Galactic [N II] line. The spectrum is averaged over the entire field of view for which order 136 is transmitted. The long wavelength cutoff occurs because the etalon gap was set so that the on-axis wavelength was $\sim 6593 \AA$. (b) Spectrum for order 135. (c) Blocking filter transmission percentage as a function of wavelength, normalized to the peak transmission. The transmission is averaged over the same region as for the spectrum shown in b). 


\section{REDUCTIONS}

Due to the narrow effective bandpass, low surface brightness and relatively small solid angle over which we expect a signal, we are severely shot-noise limited and in addition must deal with a number of subtle systematic effects which further degrade the signal. Therefore, in addition to the observing procedure described above, the reduction of the data must deal as well as possible with these difficulties and hence we describe in some detail various steps in the reduction of the data. We first give a simple overview of the basic reduction procedure and the approximations implicit in it for both the flux calibration observations and the target itself.

\subsection{Basic equations}

The count rate in ADU s ${ }^{-1}$ expected from a very small patch of sky (e.g., a pixel corresponding to solid angle $\Delta \Omega$ ) may be expressed as

$$
(d C / d \Omega) \Delta \Omega=g \epsilon\left(\lambda_{\max }(\Omega)\right) A_{e f f}\left(\Omega, \lambda_{\max }(\Omega)\right) \int_{-\infty}^{+\infty} S\left(\lambda^{\prime}, \Omega\right) R\left(\lambda^{\prime}-\lambda_{\max }(\Omega)\right) d \lambda^{\prime}
$$

In this expression $S(\lambda, \Omega)$ is the photon surface brightness per $\AA$ as a function of wavelength and direction. At any given point on the detector, the Fabry-Perot will have its maximum response at some wavelength $\lambda_{\max }(\Omega)$, and at this wavelength and direction we denote the overall effective collecting area of the system as $A_{\text {eff }}\left(\Omega, \lambda_{\text {max }}(\Omega)\right.$ ) (not including the atmospheric attenuation, $\epsilon(\lambda)$ and the CCD gain in $\mathrm{ADU} \mathrm{e}^{-1}, g$, which are included separately in equation (2)). This effective area includes the spatial and wavelength dependence of the blocking filter and the pixel-to-pixel sensitivity of the CCD, determined via flat field images, as described below in $§ 3.2$. However, the etalon will have some response in this direction to 
photons differing from $\lambda_{\max }(\Omega)$ and we denote this response function relative to its maximum response by $R\left(\lambda-\lambda_{\max }(\Omega)\right)$. In principle, as in any system with finite spatial and wavelength resolution, we would need to invert equation (2) to reconstruct the actual distribution of surface brightness, $S(\lambda, \Omega)$.

In practice, spectra for both the standard star and the target are constructed as follows. 1) We define a fine grid in wavelength with a uniform spacing, $\Delta \lambda_{\text {grid }}$ of $0.11 \AA$. 2) For each pixel within the target mask (described below) we find the wavelength $\lambda_{\max }$ associated with the center of that pixel, calibrated using the series of neon lamp observations described below. 3) We associate all the counts in that pixel with the wavelength bin in this fine grid closest to $\lambda_{\max }$. After extraction on this $0.11 \AA$ interval grid, the spectra were then binned on a coarser $0.34 \AA$ grid, and all plots, fits and analyses of the spectra use the $0.34 \AA$ grid. We refer hereafter to each element of this coarser grid as a "wavelength bin" to distinguish it from the spatial pixels.

Consider first the observations of an early type standard star for calibration of the throughput of the system. In this case, over the wavelength region of interest the flux is constant with wavelength and the spatial structure is simply the seeing disk. In this case, the total count rate from the star will be

$$
C_{s t d}=g \epsilon\left(\lambda_{\max }(\Omega)\right) A_{e f f}\left(\lambda_{\max }(\Omega)\right)[d N / d \lambda]_{s t d} \Delta \lambda_{E B}
$$

In this expression, $[d N / d \lambda]_{s t d}$ is the (known) monochromatic flux (in photons $\mathrm{cm}^{-2} \mathrm{~s}^{-1}$ $\AA^{-1}$ ), from which the effective area at this wavelength and this location on the detector may be calculated. The quantity $\Delta \lambda_{E B}$ is the effective bandwidth of the etalon, which is the response function $R\left(\lambda-\lambda_{\max }\right)$ (equation 2) integrated over $\lambda$. The response function of the Fabry-Perot is quite accurately represented by a Voigt profile, and we determine the effective bandwidth empirically using neon comparison lines. When the procedure described 
above is applied to an unresolved continuum object, the only error is simply that associated with the aliasing of a detector which is not adequately sampled if the intra-pixel response is not uniform; however, this effect can be minimized by small dithering of the standard star observations.

For the observation of the target, the opposite situation holds: the spatial scale is much larger than the pixel size, but the expected wavelength dependence in the neighborhood of the emission line is, in our case, comparable to the etalon resolution. In this case, the count rate from the source if it were finely sampled spatially can be shown to be just the convolution of the intrinsic emission line profile with the etalon response:

$$
F\left(\lambda, \lambda_{0}\right)=g \in A_{e f f} \quad B_{0} \int_{-\infty}^{+\infty} G\left(\lambda^{\prime}-\lambda_{0}\right) R\left(\lambda^{\prime}-\lambda\right) d \lambda^{\prime}
$$

where $B_{o}$ is the wavelength-integrated photon surface brightness, and $G$ is the normalized intrinsic line profile. We have omitted the angular dependence for clarity. The instrumental profile $R$ is a Voigt function, and as discussed in $§ 3.2$ the convolution represented by the integral in equation (4) results in an observed profile which is also well described by a Voigt function. Then the photon surface brightness $B_{o}$ is obtained from the count rate summed over the line profile $F^{\prime}=\sum_{i} F_{i} \Delta \lambda_{\text {grid }}$ as

$$
B_{o}=\frac{F^{\prime}}{g \epsilon A_{e f f} \Delta \lambda_{E B}}
$$

The effective area $A_{\text {eff }}=\eta A$, where $A$ is the telescope area and $\eta$ is the efficiency of the entire optical path including telescope and instrument optics, blocking filter, etalon, and CCD. Using the standard star observations, we determined that $\eta=0.22$. Photon rates were converted to surface brightness, corrected for the small amount of Galactic extinction, which from Schlegel, Finkbeiner, \& Davis (1998) amounts to about 0.05 mag in the R band. 
In practice, as in conventional spectrographs, if the sampling due to the pixel size is not adequate, there may be aliasing of the spectrum. For the etalon order nearest the optical axis of the etalon, the sampling is adequate in spite of the $4 \times 4$ binning. However for the remaining orders this is no longer the case. If each wavelength bin were only sampled once, this could result in an emission line profile whose center and width were poorly defined. In fact, this situation is entirely mitigated because the format produced by the etalon causes each bin in the wavelength grid to lie at more-or-less random positions on the more than 100 pixels contributing to each wavelength bin, just as "dithering" does in a conventional undersampled spectrograph.

\subsection{Details of the Reduction Procedure}

The first steps in the data reduction for all calibration and data CCD frames were bias and dark frame subtraction; bias and dark frames were constructed from the median value for each pixel derived from stacks of bias and dark frames in order to minimize the noise contribution from this step. Next, spatial variations in the instrument and CCD response were removed by dividing by a normalized flat-field frame obtained from a stack of exposures of the standard flat-field lamp illuminating a screen on the side of the dome. We used a flatfield frame obtained at the same etalon gap spacing $l$ as used for the observation; we masked pixels for which the flat-field response was less than $60 \%$ of the peak.

The wavelength response given by equation (1) assumes ideal optics. In practice, there are deviations that result in slight shifts in the response; for optimum resolution we characterize these deviations as follows. We illuminate the dome screen with a neon lamp, stepping the etalon gap over a free spectral range in steps of half the wavelength resolution. Then at each pixel we determine the etalon gap that maximally transmits the neon calibration line. In this way, we characterize the deviations from the ideal wavelength relation given by 
equation (1).

As mentioned in $\S 2.2$, the standard method to monitor and calibrate temporal variations is to take periodic exposures of a diffuse monochromatic calibration lamp. Because we have bright night sky lines of known wavelength in each exposure, it is more accurate and efficient to use these. In principle, we could determine the centroid and radius of one of the night sky rings; however, in practice stars and cosmic rays make this difficult. Instead, we used the following method. The wavelength error caused by an incorrect choice of optical axis and etalon gap varies as a function of position in the field. The night sky lines present in the field (e.g., Fig. 1) have known wavelengths. We compare the known wavelengths with those computed for a large range of assumed optical axes and etalon gap, and select the axis and gap which yield the smallest difference between the calculated and known wavelengths.

We used the Tukey biweight statistic as described by Beers, Flynn, \& Gebhardt (1990) to estimate the count rate per pixel for each wavelength bin based on all the pixels contributing to that wavelength bin. As described by Beers, Flynn, \& Gebhardt (1990), the biweight estimator is more resistant against outliers and more robust than the mean, and more efficient than the median. It is highly effective at minimizing the effects of cosmic rays and stars.

To check that variations across the frame do not cause offsets between the H $\alpha$ part of the spectrum and the rest of the spectrum, we divided the frame into several regions and compared the spectra extracted from each region. Differences in these spectra were small, and are dominated by scattered light from stars; this effect is described next.

Even though spectral features in stars in the field are likely to be weak, these stars can nonetheless create spectral artifacts. Recall that the count rate in each wavelength bin is determined from the biweight of the count rate for the CCD pixels corresponding to that wavelength bin. Although stars are effectively rejected by the biweight estimator, which excludes pixels with count rates that deviate by more than four standard deviations from 
the central location, scattered light from brighter stars can cause difficulties. This is because portions of the extended skirts of the point spread function can be too weak to be rejected by the biweight estimator, yet extend over a sufficiently large number of pixels so as to bias the biweight.

Accordingly, we adopted the following procedure. First, we excluded regions for which scattered stellar light was detectable by visual inspection of the frames. Since the error introduced by a star increases with the size of the region that escapes the biweight cutoff, and since the larger regions are of course associated with the brightest stars, this was straightforward, and in itself greatly reduced much of the problem associated with star light. This eliminated artifacts in individual spectra that were as large as $5 \times 10^{-20} \mathrm{ergs} \mathrm{cm}^{-2} \mathrm{~s}^{-1} \operatorname{arcsec}^{-2} \AA^{-1}$, although making a difference of less than $5 \times 10^{-21} \mathrm{ergs} \mathrm{cm}^{-2} \mathrm{~s}^{-1} \operatorname{arcsec}^{-2} \AA^{-1}$ to the final averaged spectrum. As an added precaution, we identified the remaining stars in the field using the program SExtractor (Bertin \& Arnouts 1996). Next, using a point spread profile determined on brighter stars, we subtracted the scattered light contribution from the remaining stars; the resulting changes to the spectra were minor.

As described in $§ 3.3$, the incident ionizing flux is inferred from the $\mathrm{H} \alpha$ brightness toward regions with observed column densities $N(H I)>10^{19} \mathrm{~cm}^{-2}$ using the $75^{\prime \prime} \mathrm{H}$ I maps provided in digital form by Chengalur, Giovanelli \& Haynes (1995). Therefore, we excluded regions with lower H I column densities in constructing the spectrum for the wavelength interval expected for the redshifted $\mathrm{H} \alpha$ emission, $6588.9 \AA<\lambda<6591.9 \AA$. Because pixels with wavelengths outside this range were not excluded, the number of pixels per wavelength bin over the wavelength interval defining the continuum was a factor of two to three higher than for the region of line emission.

In Figure 3 we show the image resulting from directly adding the GH1 and GH2 frames of one W-GH1-GH2-E set and subtracting the $\mathrm{W}$ and $\mathrm{E}$ frames. The $\mathrm{OH}$ night sky lines 
largely cancel, while the [N II] line appears black, corresponding to brighter [N II] emission in the GH1 and GH2 frames than the sky frames. Note the patchy spatial distribution of the [N II] emission and the presence of two well defined velocity components. Also note that the $\mathrm{OH}$ line residuals appear to have a slight offset in the subtracted image. This occurs because of drifts in the etalon. Because of such drifts, spectra are extracted not from W-GH1-GH2-E combined frames such as shown here, but from the individual frames because drifts in the wavelength scale can be corrected during the extraction process, as described above. The ring corresponding to the approximate location of the expected redshifted $\mathrm{H} \alpha$ signal with centroid at $6590.4 \AA$ is shown by the solid-line circle.

Fig. 3.- Target minus sky frame produced from a single W-GH1-GH2-E set. Several OH and $[\mathrm{N} \mathrm{II}]$ features are marked. Spatial variations in the Galactic [N II] features are evident, corresponding to differences in $[\mathrm{N} \mathrm{II}]$ emission velocity and spatial distribution between the target and sky fields, along with $\mathrm{OH}$ night sky residuals. Also indicated are the $N(H)=10^{19}$ $\mathrm{cm}^{-2}$ contour for the southwest HI $1225+01$ cloud (GH SW) and for the bridge between the SW and NE components; the H I data are from Chengalur, Giovanelli \& Haynes (1995). The cross marks the etalon optical axis. The solid circle shows the locus of $6590.4 \AA$ in order 136, the expected wavelength for $\mathrm{H} \alpha$, while the dashed arc shows the same locus for order 135, where little or no $\mathrm{H} \alpha$ is expected.

Target minus sky (i.e. On-Off) spectra produced for orders 136 and 135 from each W-GH1-GH2-E set of four spectra are shown in Figures 4 and 5; each On-Off spectrum corresponds to 30 minutes integration time on the target. The error bars shown on the bottom spectrum of Figures 4 and 5 are calculated from Poisson statistics; the errors are larger at the wavelengths of night sky lines due to the larger count rates, and in the wavelength range $6588.9 \AA<\lambda<6591.9 \AA$ due to the smaller number of unmasked pixels. Significant non-zero residuals are seen at the wavelengths of the Galactic [N II] line; this is due to the 
difference in high Galactic latitude [N II] emission between the on and off positions. Also the atmospheric lines and the continuum level typically show non-zero residuals due to temporal and spatial variations in night sky brightness.

Figure $6 \mathrm{a}$ is reproduced from Figure $2 \mathrm{~b}$ to enable comparison with Figure $6 \mathrm{~b}$ which shows the target minus sky spectra for orders 135 and 136 averaged over the 15 W-GH1GH2-E spectra obtained during the run. In Figure 6c, we show a magnified view of the spectra, including error bars. The error bars in Figure 6c are estimated from the scatter at each wavelength between the 15 spectra that contribute to the average and have decreased by approximately a factor of four from the individual W-GH1-GH2-E spectra, as expected. Small negative residuals remain at the $\mathrm{OH}$ wavelengths, at a level of about $1 \%$ of the line strength, due to nonlinear temporal variations in the $\mathrm{OH}$ line strength.

There is also a non-zero residual continuum level. We determined this residual continuum level (assumed constant with wavelength over this narrow range) by averaging over 6598.4 $\AA<\lambda<6601.5 \AA$ and $6606.3 \AA<\lambda<6610.8 \AA$ for order 135. The residual continuum level is $1.4 \times 10^{-20} \mathrm{ergs} \mathrm{cm}^{-2} \mathrm{~s}^{-1} \operatorname{arcsec}^{-2} \AA^{-1}$, about $0.3 \%$ of the continuum brightness. As expected the percentage continuum residual is smaller than the $\mathrm{OH}$ residual since the $\mathrm{OH}$ lines are intrinsically more variable.

There is a weak $\mathrm{OH}$ line at $6591.7 \AA$ evident in Figure $6 \mathrm{a}$ and which also has been identified by Osterbrock et al. from compilation of Keck spectra (Osterbrock, Fulbright, $\&$ Bida 1997). We fit each of the four $\mathrm{OH}$ lines in Figure 6a to determine the ratios of the $\mathrm{OH}$ line intensities. The $6591.7 \AA$ line is about $10 \%$ of the strength of the $6596 \AA$ line, similar to what can be derived from the Osterbrock, Fulbright, \& Bida (1997) Keck data. Based on the residual negative $\mathrm{OH}$ emission of the bright $\mathrm{OH}$ line at $6596 \AA$ of about $2 \times 10^{-20} \operatorname{ergs} \mathrm{cm}^{-2} \mathrm{~s}^{-1} \operatorname{arcsec}^{-2} \AA^{-1}$, we expect a peak residual of approximately $-2 \times 10^{-21} \operatorname{ergs~cm}^{-2} \mathrm{~s}^{-1} \operatorname{arcsec}^{-2} \AA^{-1}$ for the 6591.7 line. We fit the residual fluxes in each 


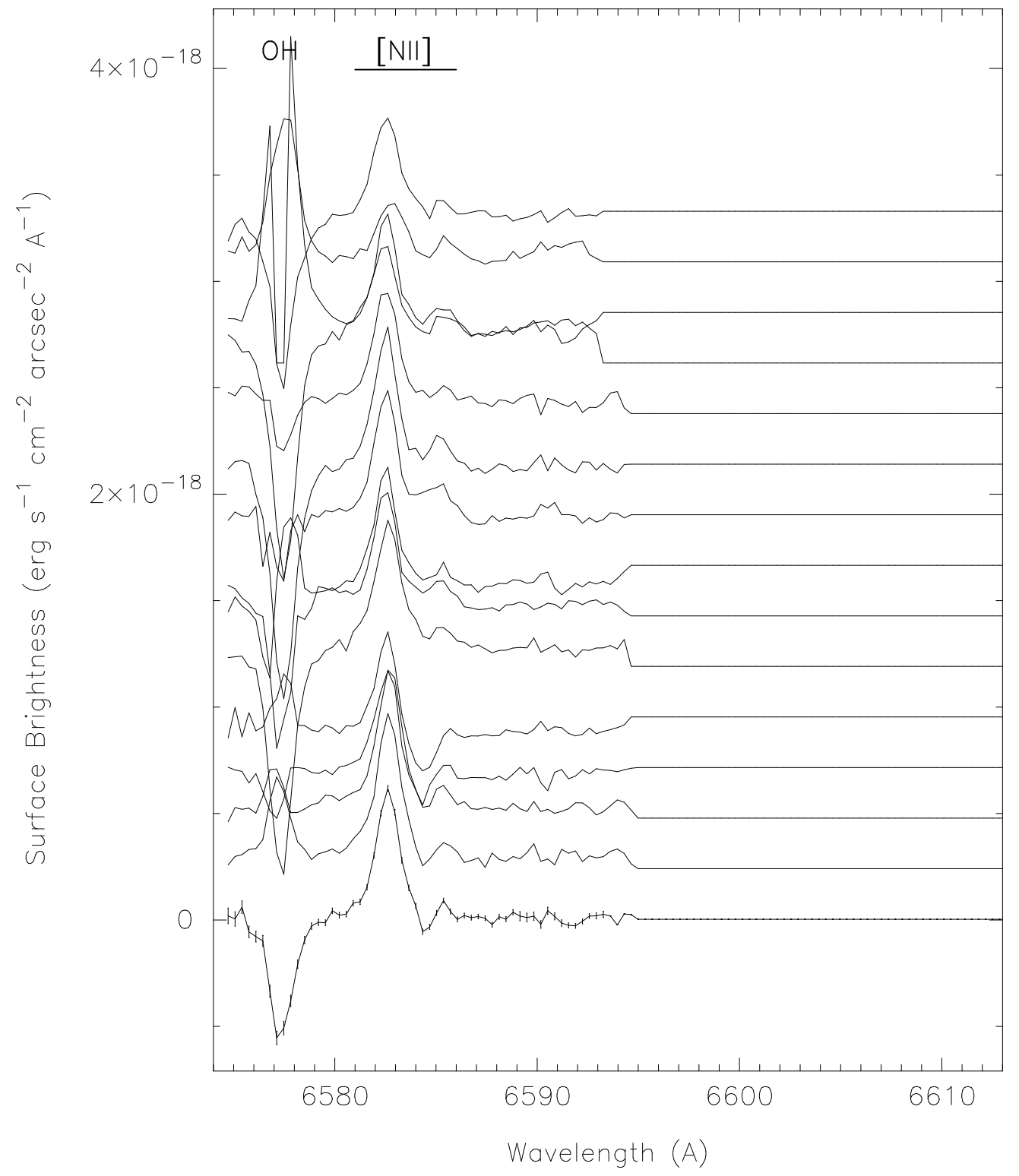

Fig. 4.- Target minus sky spectra for each of the 15 W-GH1-GH2-E sets obtained during the run are shown for order 136. The error bars shown on the bottom spectrum are calculated from Poisson statistics. 


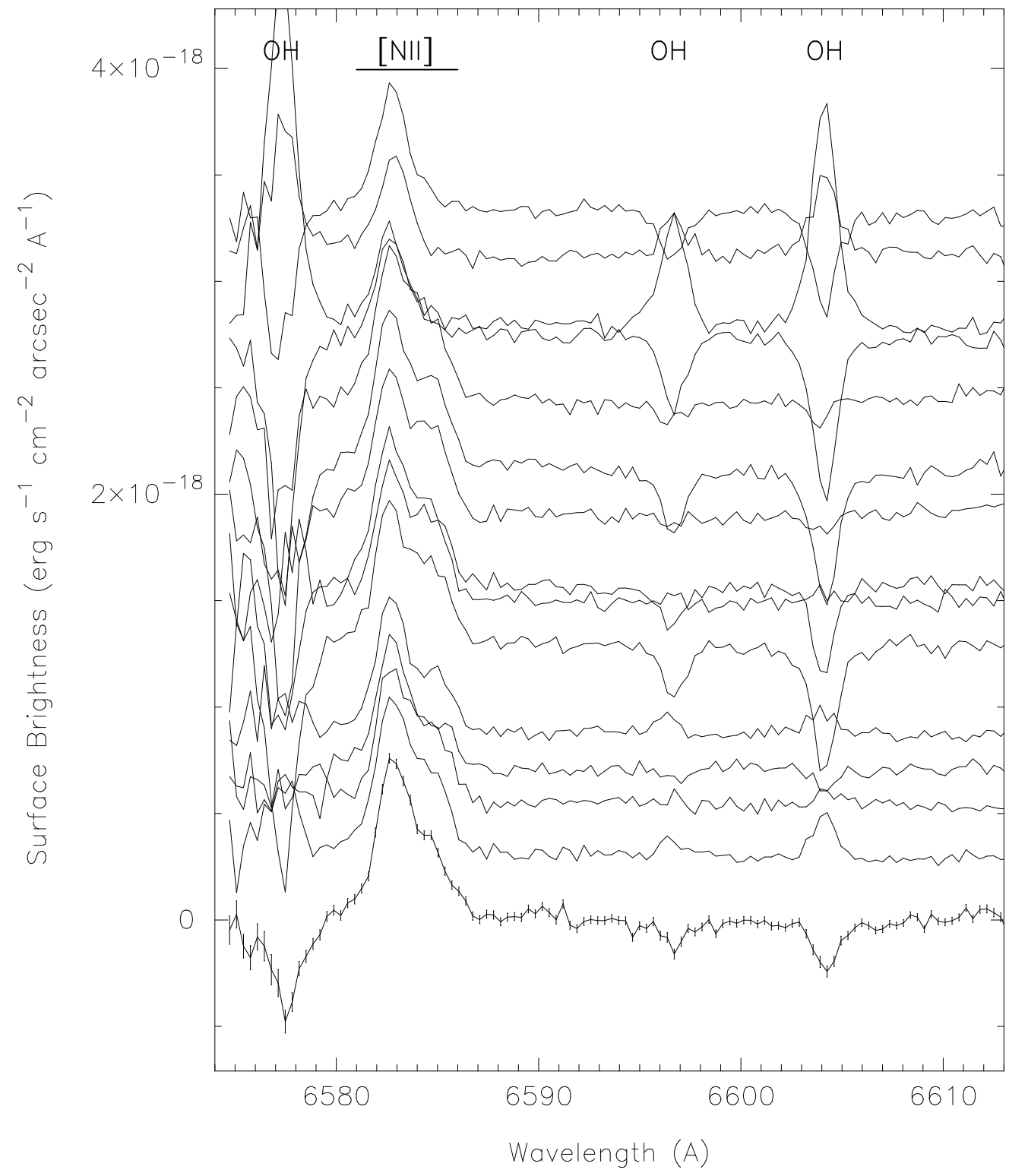

Fig. 5.- Target minus sky spectra for each of the 15 W-GH1-GH2-E sets obtained during the run are shown for order 135. The error bars shown on the bottom spectrum are calculated from Poisson statistics. 


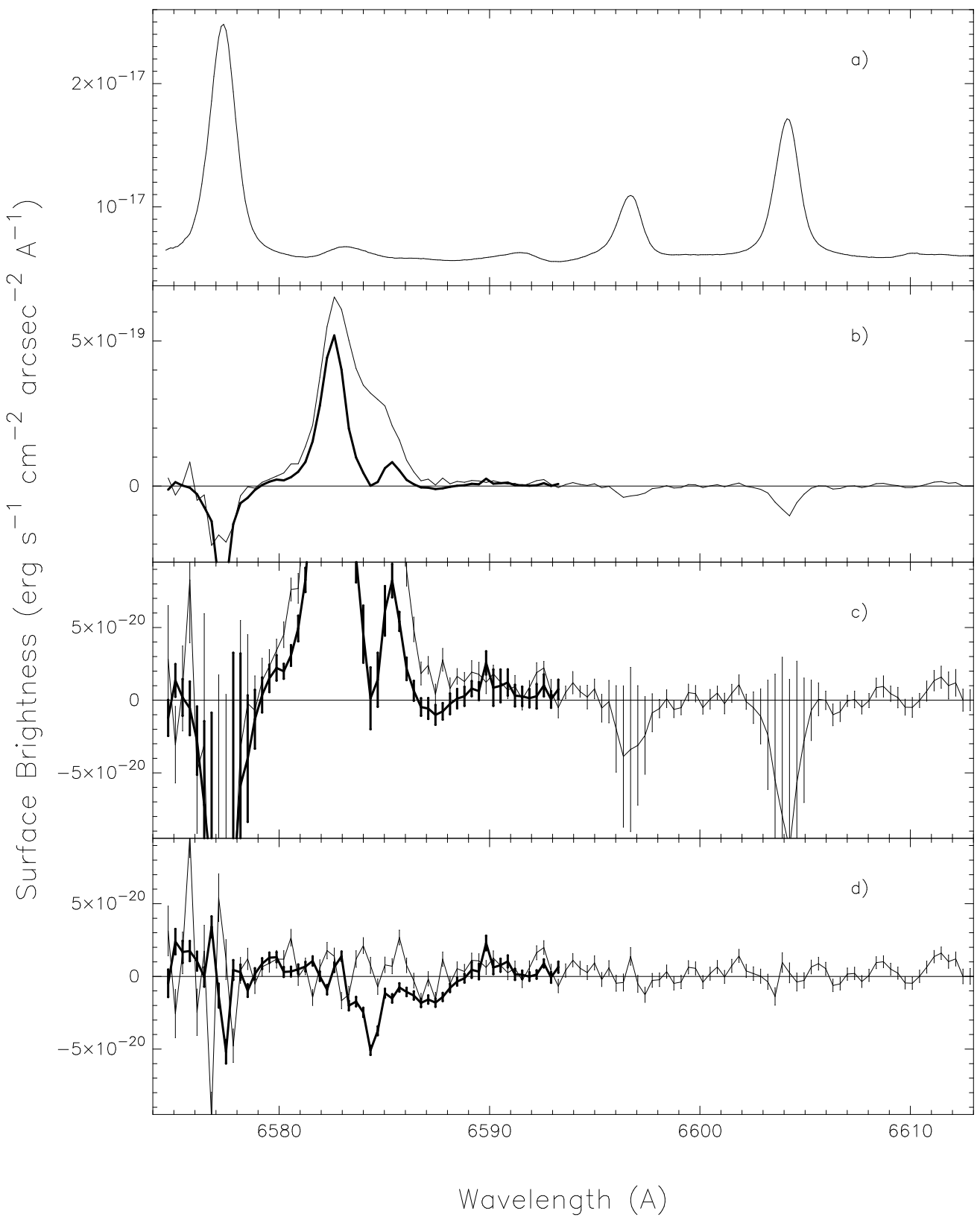

Fig. 6.- (a) Same spectrum as in Figure $2 \mathrm{~b}$ showing the lines in the bandpass. (b) The average of all target minus sky spectra for order 136 (thick line) and 135 (thin line). Multiple components in the residual [N II] line are evident, due to differences in the spatial distribution of [N II] between the target and sky positions. (c) Expanded view of spectra shown in the panel above. Error bars are $1 \sigma$ derived from the scatter of the $15 \mathrm{~W}-\mathrm{GH} 1-\mathrm{GH} 2-\mathrm{E}$ spectra. (d) The same spectra as in (c), except the continuum, OH, and [N II] residuals have been subtracted as described in the text. Here the error bars are those calculated from Poisson statistics. 
of the $\mathrm{OH}$ lines shown in Figures $6 \mathrm{~b}$ and 6c using a Voigt function; for the fit, the Gaussian and Lorentzian line width parameters were held fixed using values determined from a fit to the neon calibration line. Also, the wavelength was fixed at the known value of the $\mathrm{OH}$ lines, and the continuum was fixed at zero since the fits were made to continuum subtracted data.

The residual $[\mathrm{N} \mathrm{II}]$ peaks in Figure $6 \mathrm{~b}$ are at $-50 \mathrm{~km} \mathrm{~s}^{-1}$ and $+80 \mathrm{~km} \mathrm{~s}^{-1}$ respectively. The brightness of this [N II] feature is close to the detection threshold of the observations of known high velocity clouds made with the same instrument (Weiner, Vogel, \& Williams 2000), but with the sensitivity of the present observations it is a highly significant detection and clearly not an artifact. The brightness of the $80 \mathrm{~km} \mathrm{~s}^{-1}$ feature varies significantly on scales of roughly an arc minute, and arcs corresponding to this feature can even be seen in a few locations in Figure 1.

In attempting to subtract off the [N II] from the spectrum it is necessary to model the feature with both components due to the broad Voigt wings in the etalon point spread function. Unfortunately, however, the strength and extent in velocity space of this [N II] makes the locating of the continuum around the expected position of the redshifted $\mathrm{H} \alpha$ more difficult, since the $[\mathrm{N} \mathrm{II}]$ emission is clearly present to within $\sim 140 \mathrm{~km} \mathrm{~s}^{-1}$ of the $\mathrm{H} \alpha$ and conceivably could extend to greater velocities at lower intensity. Moreover, the clear differences in structure of the [N II] between the on and off positions makes the onoff sky subtraction problematic over the spectral region around the $[\mathrm{N}$ II]. Note that the [N II] spectra even over the relatively small angles separating orders 136 and 135 are slightly different due to spatial variations in the [N II]. These can readily be seen by direct inspection of the difference images (e.g., Figure 3).

Although the presence of the [N II] adversely affects the measurement of the $\mathrm{H} \alpha$ signal which is our primary objective, it nonetheless is an interesting feature in itself. We are not aware of other observations in this general part of the sky that would have the sensitivity 
to detect $\mathrm{H} \alpha$ or $[\mathrm{N} \mathrm{II}]$ emission at the level we detect. In $\mathrm{H}$ I, the Leiden-Dwingeloo survey (Burton \& Hartmann 1994) clearly detects emission only in the range $-50<V_{L S R}<+40$ $\mathrm{km} \mathrm{s}^{-1}$. A similar result is found in the HIPASS survey (Barnes et al. 2001). Thus, we cannot at present associate the $80 \mathrm{~km} \mathrm{~s}^{-1}$ [N II] emission with known H I emission. The velocity of the $80 \mathrm{~km} \mathrm{~s}^{-1}$ feature is nearly large enough to qualify as a high velocity cloud (Wakker \& van Woerden 1997); however, Wakker \& van Woerden (1997) emphasize that the deviation velocity (the difference from the largest velocity permitted by Galactic rotation) is a better criterion, arguing for a deviation velocity greater than $50 \mathrm{~km} \mathrm{~s}^{-1}$ as the cutoff. Using the model presented by Wakker (1990), positive velocities due to rotation are not expected in this direction due to its high Galactic latitude $\left(b=64^{\circ}\right)$; consequently the deviation velocity is $80 \mathrm{~km} \mathrm{~s}^{-1}$, suggesting that the detected emission may be produced by a high-velocity cloud. With an emission measure of $25 \mathrm{mR}$, the [N II] emission is comparable to the weakest H $\alpha$ emission from known high velocity clouds observed by Weiner, Vogel, \& Williams (2000); however, [N II] emission was not detected by Weiner, Vogel, \& Williams (2000) toward the faintest high velocity clouds, with upper limits somewhat below our detection. In other words the [N II] emission we detect is somewhat brighter than in some known $\mathrm{H}$ I high velocity clouds, in spite of the fact that no $\mathrm{H} \mathrm{I}$ emission is seen in this direction at this velocity. The detected [N II] emission is clearly interesting and merits further study to determine its origin.

The resulting spectra with the continuum, $\mathrm{OH}$ line, and [N II] line residuals subtracted are shown in Figure 6d. In this figure, the error bars have been calculated from Poisson statistics. Note that the order 135 and 136 spectra are generally the same within the statistical errors, except for part of the $[\mathrm{N}$ II] line; this is due to spatial structure in the $[\mathrm{N}$ II] distribution and the fact that the difference in [N II] distribution between the target and sky positions must be slightly more complex than can be modeled with just two components. 
Next we fit a Voigt profile to this spectrum over the wavelength interval $6588 \AA<\lambda<$ $6593.5 \AA$ (corresponding to about four spectral resolution elements). The continuum is fixed to be zero since the continuum has been subtracted, as discussed above. The instrumental profile is taken from the neon calibration.

The assumed intrinsic $\mathrm{H} \alpha$ line profile was obtained as follows. Using the $75^{\prime \prime}$ resolution VLA H I observations of Chengalur, Giovanelli \& Haynes (1995) (which are given in the heliocentric frame), we determined the H I velocities of the gas at each pixel on the FabryPerot ring at the approximate redshifted wavelength of $\mathrm{H} \alpha$ and then shifted these velocities by $+5.8 \mathrm{~km} \mathrm{~s}^{-1}$, which puts them in the barycentric frame which we have used throughout this work. Weighting each pixel equally as a first order approximation since the $\mathrm{H} \alpha$ emission is only weakly dependent on H I column density for the observed column density range, we constructed the spectrum shown in Figure 7a. H I emission occurs mostly at two velocities because HI 1225+01 has a rotation curve characteristic of a galactic disk, and the 6590.4 $\AA$ ring has relatively little overlap with the central part or minor axis (e.g., Fig. 3). This profile was then convolved with a Gaussian with a dispersion of $6 \mathrm{~km} \mathrm{~s}^{-1}$ (corresponding to a typical dwarf dispersion and slightly less than the velocity dispersion of $8 \mathrm{~km} \mathrm{~s}^{-1}$ of the NE component) and also with a Gaussian whose width corresponds to the thermal velocity of the ionized hydrogen at a temperture of $10000 \mathrm{~K}$. This produces the profile shown in Figure 7b. Finally, this profile was convolved with the instrumental profile to produce the profile shown in Figure 7c. This corresponds to the profile of the $\mathrm{H} \alpha$ line we expect to observe from HI $1225+01$. This is well fit by a Voigt profile centered at $6590.4 \AA$ and FWHM $79 \mathrm{~km} \mathrm{~s}^{-1}$.

A Voigt profile with this shape is then used as the line profile in a fit to the observed spectrum, in which the only free parameter is the Voigt line strength. The best fit line brightness is $4.2 \mathrm{mR}$. The spectrum and the $4.2 \mathrm{mR}$ best fit for the $\mathrm{OH}$, [NII], and continuum subtracted spectrum for order 136 are shown in Figure 8b. To estimate the uncertainty in 


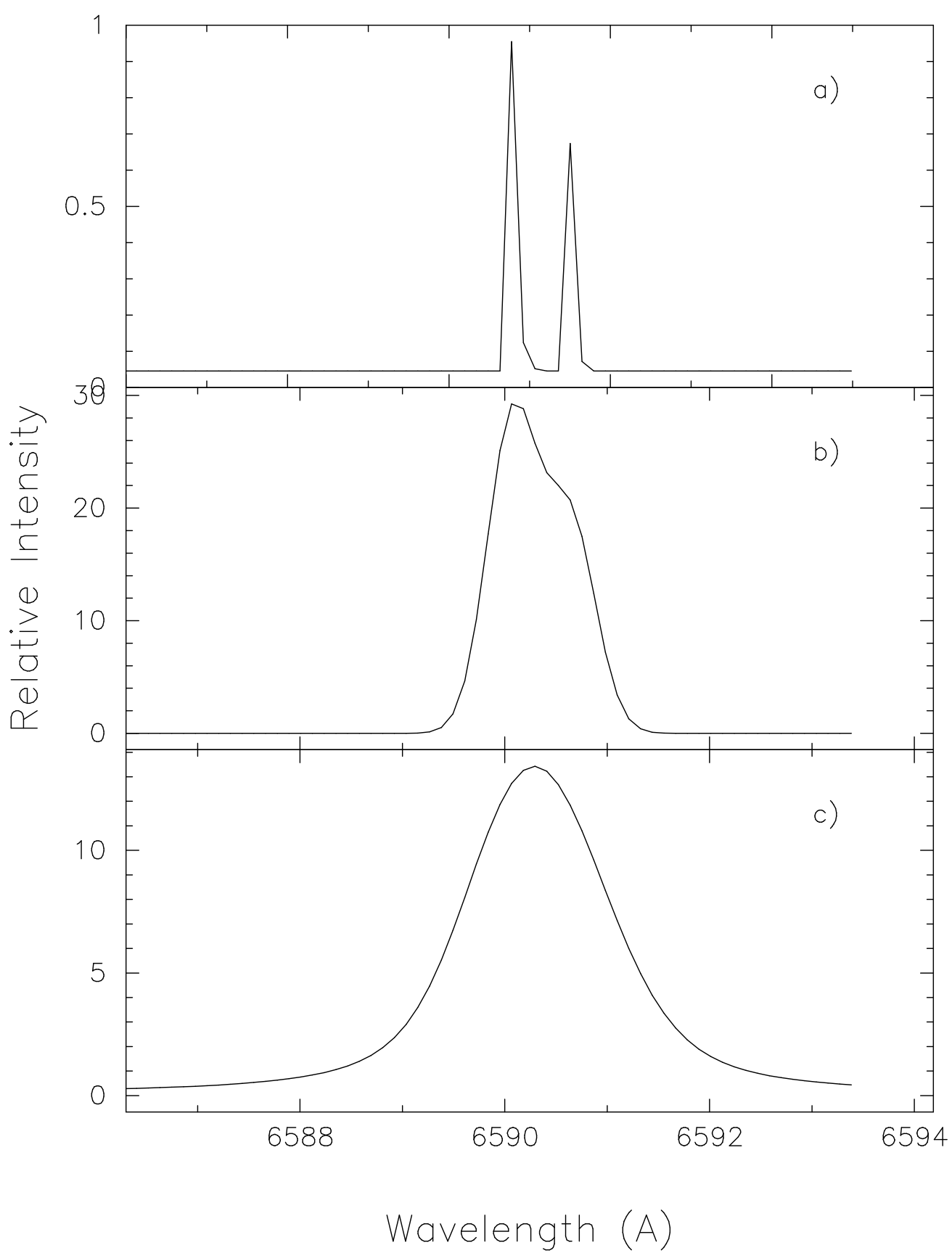

Fig. 7.- (a) Wavelength distribution of redshifted $\mathrm{H} \alpha$ centroids expected based on 75" VLA maps of H I obtained by Chengalur, Giovanelli \& Haynes (1995) for $\mathrm{H} \alpha$ emission regions observed with the Fabry-Perot. (b) Expected intrinsic $\mathrm{H} \alpha$ profile obtained by convolving the H I profile in (a) with Gaussians for $\mathrm{H}$ atoms at $10000 \mathrm{~K}$ and the $\mathrm{H}$ I velocity dispersion. (c) Expected observed $\mathrm{H} \alpha$ profile, obtained by convolving the intrinsic $\mathrm{H} \alpha$ profile in (b) with the Fabry-Perot instrumental profile. 
the line strength, we used a Monte Carlo approach (Weiner 2001), in which we constructed spectra using the errors estimated from the comparison of the individual W-GH1-GH2-E spectra (i.e. the errors shown in Figure 6c). The resulting $1 \sigma$ error bars are $\pm 1.6 \mathrm{mR}$, and the $2 \sigma$ upper limit is $7.4 \mathrm{mR}$.

Although we have attempted to accurately apply flatfield corrections to the data, differences in the spectral and spatial properties between the flatfield lamp and the sky data, as well as differences between the resolution in orders 135 and 136 mean that direct subtraction of the "on-only" data in order 135 from 136 to obtain the net signal in the region of the expected $\mathrm{H} \alpha$ signature may well mask the signal we are attempting to detect. For this reason we have employed the on-off chopping described previously, which should accurately remove the effects of these differences in the instrumental response.

However, although the sequence of on-off observations removes these differences as well as temporal variations in the night sky background which are linear, the characteristic time for the full cycle for one off-on-on-off sequence is over an hour, and variations over a shorter time may well occur. Therefore the order 135 spectrum of Figure 6d was subtracted from order 136 in the same figure to produce Figure 8c. (In order to reduce the noise in this subtraction the data in order 135 were first smoothed using a 5-point running mean.)

Disappointingly, the signal present in Figure 8b appears to have largely disappeared. It is known that other weak night sky emission features are present. In particular, weak emission from $\mathrm{O}_{2}$ has been found in the night sky spectrum (Slanger et al. 2000) and one of these emission features falls at the expected wavelength of $\mathrm{H} \alpha$ (T. Slanger 2001, private communication). No attempt has been made to model any residual emission from this source. However, the impression is that the main effect of the subtraction of 135 from 136 is simply to lower the level of the continuum so that it falls below the nominal zero level. This impression is confirmed by again fitting a Voigt profile to the same data as shown in Figure 
8c, but now allowing the continuum level to be a second free parameter. The resulting best fit, shown in Figure 8d, yields $3.9 \mathrm{mR}$, very similar to that found above. Because of the very limited continuum region in order 136, which is affected nearly up to the $\mathrm{H} \alpha$ signal at shorter wavelengths by the [NII], as described above, and only extends to $\sim 6593 \AA$ which is the termination of order 136 as explained in $\S 2.2$, it is not clear whether such an offset really exists.

It is also not clear what could give rise to such a continuum offset. Spatial gradients in the background continuum arising from the zodiacal light and airglow continuum ${ }^{1}$ would have to be present over an extent of only $\sim 8^{\prime}$. It should be borne in mind however that the possible offset is only about $0.1 \%$ of the total continuum level. The data provide no evidence for such gradients; first, the CCD dewar was rotated $180^{\circ}$ after the second night, yet comparison of spectra obtained from the opposite rotations does not show mirrored spectra artifacts; second, comparison of the order 135 and 134 spectra in Figure 9d reveals no significant difference, even though these are extracted from regions offset by $\sim 5^{\prime}$, nearly as large as the $7-10^{\prime}$ offset between orders 136 and 135 .

A more likely possible source of artifacts is scattered light from stars that has not entirely been removed or masked. This scattered light would be more likely to create spectral artifacts in the central order. Partly this is because out of focus reflections between the etalon and blocking filter are not directed out of the beam for the central order due to the etalon tilt angle selected. Also, due to the quadratic nature of the wavelength dispersion scattered star light affects relatively narrow spectral regions in the central order compared to the outer orders. This may explain why the order 135 and 134 spectra (e.g., Figs. 8a) are quite similar, and more featureless than the order 136 spectrum.

\footnotetext{
${ }^{1}$ This airglow is generally ascribed to $\mathrm{NO}_{2}$, although there is some disagreement on this point.
} 
Although there is some weak indication of the detection of a line from Figures $8 \mathrm{~b}$ and $8 \mathrm{~d}$, with strengths of $\sim 4 \mathrm{mR}$, because of this uncertainty we cannot claim a positive direct detection of the ionizing background radiation, but the formal $2 \sigma$ upper $\operatorname{limit}^{2}$ to any signal from the fits in either $8 \mathrm{~b}$ or $8 \mathrm{~d}$ is about $8 \mathrm{mR}$, and we shall use this limit in our subsequent discussion. The actual formal upper confidence limits to the fits in Figures 8b, c, and d are shown in Figures 9b, 9c, and 9d, where Figure 8a is again reproduced for reference as Figure 9 a.

\subsection{Conversion of the $\mathrm{H} \alpha$ Surface Brightness to Ionizing Flux}

Following VWRH and Stocke et al. (1991), (see also SRGPF) the one-sided incident ionizing flux is defined as

$$
\Phi=\pi \int_{\nu_{0}}^{\infty} \frac{J_{\nu}}{h \nu} d \nu
$$

where $J_{\nu}$ is the mean intensity. The factor $\pi$ assumes that the background intensity is isotropic. From our measurement of the $\mathrm{H} \alpha$ surface brightness, $I_{H \alpha}$, we may calculate $\Phi$ from:

$$
\Phi=4 \pi \frac{I_{H \alpha}}{h \nu} \frac{1}{f_{a} f_{H \alpha}}\left(\frac{A_{\text {proj }}}{A_{t o t}}\right)
$$

which can be written as

\footnotetext{
${ }^{2}$ The Monte Carlo calculations actually lead to a direct estimate of an upper confidence limit of $97.5 \%$, which for a Gaussian distribution would imply that $95 \%$ of the Monte Carlo trials lie between $-2 \sigma$ and $+2 \sigma$ of the best fit value, but we will simply refer to this as the " $2 \sigma$ " upper limit.
} 


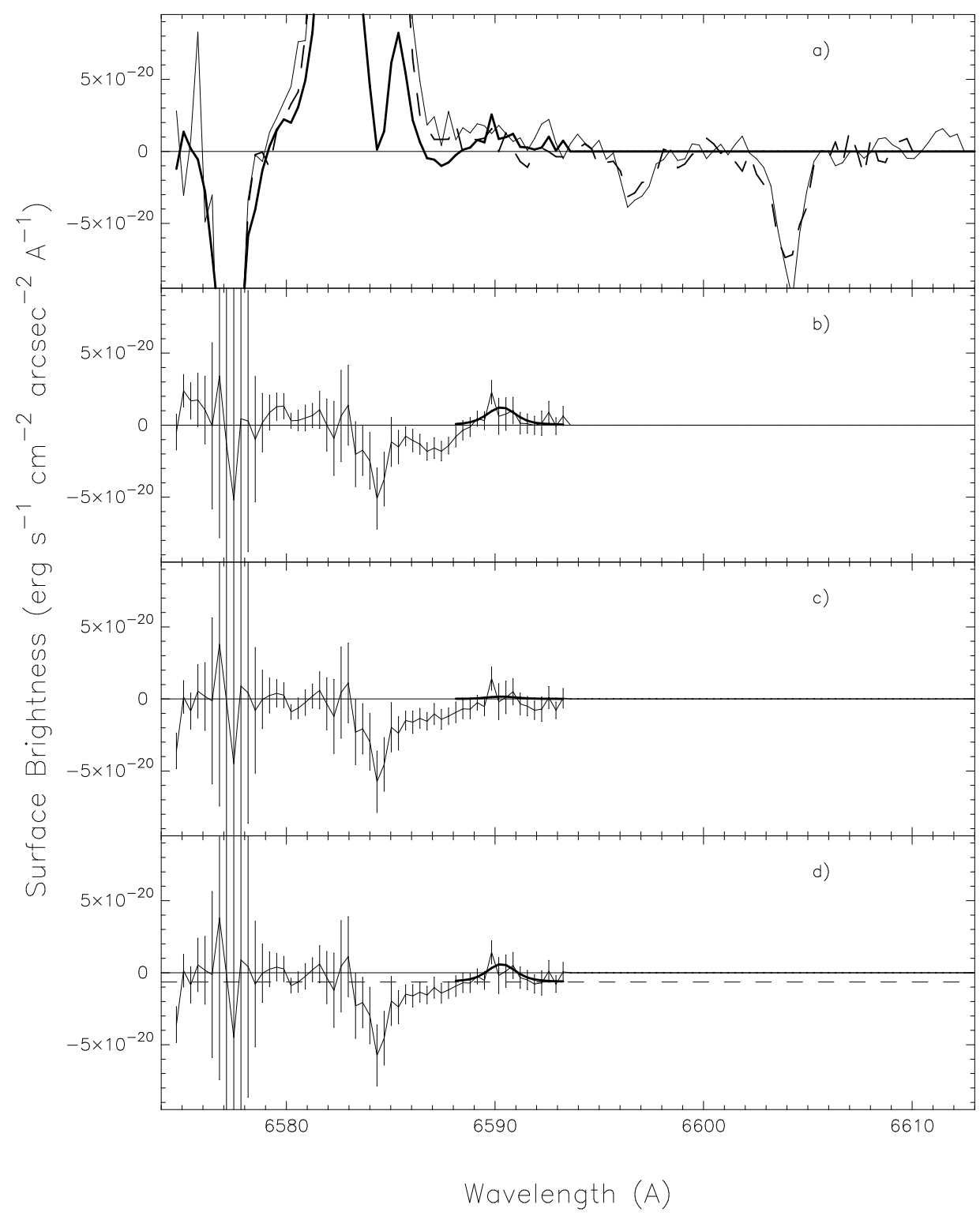

Fig. 8.- (a) Target minus sky spectra compared for orders 136 (thick solid line), 135 (thin solid line), and 134 (dashed line). In this panel, unlike those below, $\mathrm{OH}$ and [N II] residuals have not been subtracted. (b) Order 136 spectrum with continuum, OH and [N II] lines subtracted, shown as a thin line; the error bars are estimated from the data. The thick line is a fit of a Voigt profile in which the only free parameter is the line strength. The best fit emission line has strength $4.2 \pm 1.6 \mathrm{mR}$. (c) As in (b), except a smoothed order 135 spectrum has been subtracted. The Voigt fit has strength $0.8 \pm 1.6 \mathrm{mR}$. (d) As in (c) except that the fit has both line strength and continuum level as free parameters. The best fit is $3.9 \mathrm{mR} \pm 2.2 \mathrm{mR}$. The best-fit continuum is shown as a horizontal dashed line. 


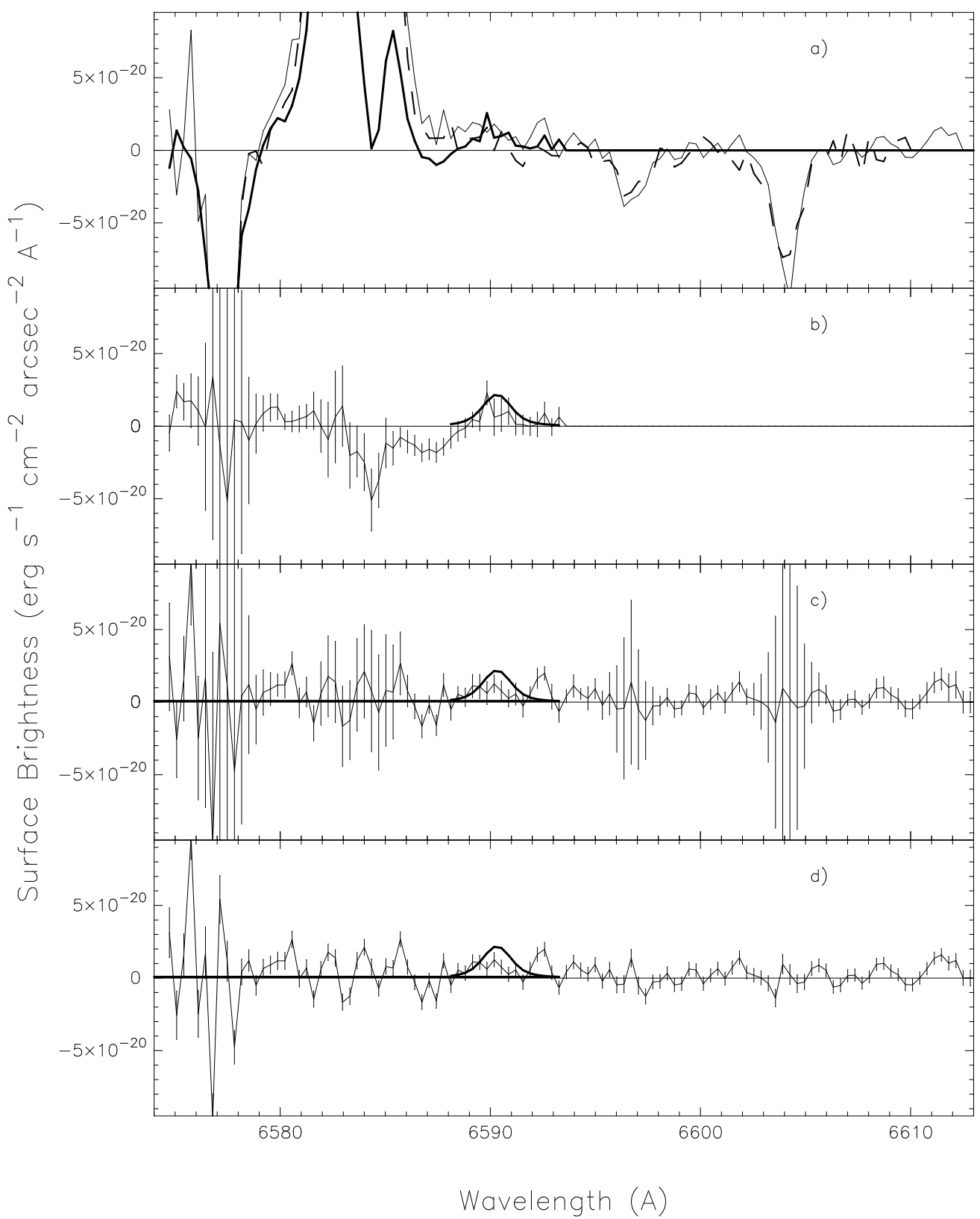

Fig. 9.- (a) Target minus sky spectra compared for orders 136 (thick solid line), 135 (thin solid line), and 134 (dashed line). In this panel, unlike those below, OH and [N II] residuals have not been subtracted. (b) Order 136 spectrum with continuum, OH and [N II] residuals subtracted, shown as a thin line; the error bars are estimated from the data. The thick line shows a $7.4 \mathrm{mR}$ emission line, corresponding to the $2 \sigma$ upper limit. (c) Order 135 spectrum, with $7.4 \mathrm{mR}$ emission line overlaid. (d) Same as (c), except the error bars are Poisson. 


$$
\Phi=\left(\begin{array}{ll}
1.0 \times 10^{3} & \mathrm{~cm}^{-2} \mathrm{~s}^{-1}
\end{array}\right)\left(\frac{I_{H \alpha}}{m R}\right) \frac{1}{f_{a} f_{H \alpha}}\left(\frac{A_{\text {proj }}}{A_{t o t}}\right)
$$

In equations $(7)$ and $(8), f_{a}$ is the fraction of the one-sided incident flux which results in recombinations to excited states, $f_{H \alpha}$ is the fraction of those recombinations which produce $\mathrm{H} \alpha$ photons, $A_{t o t}$ is the total surface area exposed to the incident radiation and $A_{\text {proj }}$ is the observed projected surface area. This expression is quite general, except that for complex geometries the appropriate averages over the cloud surface of the quantities $f_{a}$ and $f_{H \alpha}$ need to be taken, and the $I_{H \alpha}$ surface brightness is that averaged over the projected area.

\subsubsection{Effective $H \alpha$ Recombination Rate}

The $\mathrm{H} \alpha$ spectrum has been extracted from regions for which $N_{H I} \geq 10^{19} \mathrm{~cm}^{-2}$. However, as described in section 3.3.2, recent work by Chengalur, Giovanelli \& Haynes (1995) suggests that HI $1225+01 \mathrm{SW}$ is a disk which is viewed far from the normal, with an aspect ratio of $4: 1$, so that the ratio of the total area to the projected area is $\sim 8$. If for simplicity we assume plain slab geometry, then the column density normal to the disk corresponding to the $10^{19} \mathrm{~cm}^{-2}$ column density is $2.5 \times 10^{18} \mathrm{~cm}^{-2}$, corresponding to an optical depth at the Lyman limit of 15.7. Adopting the spectral index of 1.8 suggested by SRGPF we find that at this optical depth about $87 \%$ of the normally incident photon flux is absorbed within the disk. However, some of the resulting recombinations occur to the ground state, and a portion of this diffuse Lyman continuum radiation can escape from the slab after one (or more) such scatterings, and thus does not contribute to any Balmer radiation. The fraction of the recombinations which go to the ground state depends upon the electron temperature, as does the energy distribution of this recombination radiation, and as discussed below, we adopt an electron temperature of $10000 \mathrm{~K}$. Since most of the region around the Fabry-Perot $6590.4 \AA$ ring consists of $\mathrm{H}$ I column densities in excess of $10^{19} \mathrm{~cm}^{-2}$, we calculate the average 
value of $f_{a}$ by creating a histogram of column densities which contribute to the ring (after reducing the observed values by the geometric factor of four) and for each of the values of the column density in the histogram compute the value of $f_{a}$, weighting each of these values by the number of pixels contributing to that bin. Detailed calculations then yield a fraction of the normally incident photon flux which ultimately results in recombinations to excited levels of 0.88 , which is the quantity $f_{a}$ in equations (7) and (8). (Had we used a spectral index of 1.0, the number would be reduced to 0.75 . We also note that even for a normal column density 10 times lower than our minimum value the fraction of the continuum flux which results in excited state recombinations is still slightly greater than half of the 0.88 value, namely 0.42 .)

We also require the effective recombination rate for producing $\mathrm{H} \alpha$ photons, or in the formulation used in equations $(7)$ and (8), the fraction $f_{H \alpha}$ of excited state recombinations which ultimately produce an $\mathrm{H} \alpha$ photon. The value of $f_{H \alpha}$ depends, as does $f_{a}$, upon the electron temperature, and upon whether Lyman line photons readily escape following the recombinations ("case A") or scatter internally until they are ultimately converted to Balmer and higher series photons ("case B"). Since nearly all of the recombinations occur at optical depths in the Lyman lines which are very large, case B is appropriate.

In VWRH we adopted a temperature of $20000 \mathrm{~K}$ on the assumption that a hard spectrum and low metallicity would lead to an electron temperature much higher than the value of $\sim 10000 \mathrm{~K}$ usually assumed. However this assumes a balance between atomic heating and cooling mechanisms. In regions of the low density intergalactic medium producing the low column density Ly $\alpha$ forest lines, Davé \& Tripp (2001) deduce much lower temperatures $(\sim 5000 \mathrm{~K})$ due to the fact that Hubble expansion dominates over atomic cooling processes. However, these considerations may not be applicable to the material comprising HI 1225+01 where the expansion has evidently been halted, and where the particle density is probably 
much higher than that in which the low column density Ly $\alpha$ lines arise, in which case thermal equilibrium is a much better approximation. Thermal equilibrium calculations for a range of metallicities and particle densities for gas exposed to metagalactic radiation and partially shielded by neutral H I yield temperatures in the range 8000-10000 K (Wolfire 2001 private communication). However his calculations do not extend below column densities of $10^{18} \mathrm{~cm}^{-2}$ so that on the outer skin of the cloud the temperatures might be slightly higher. We adopt an electron temperature of $10000 \mathrm{~K}$. Then, for case B and an electron temperature of 10000 K from data in Osterbrock (1989) and Pengelly \& Seaton (1964) we find $f_{H \alpha}=0.45$.

\subsubsection{Cloud Geometry}

As noted previously, Chengalur, Giovanelli \& Haynes (1995) have made a detailed study of the $\mathrm{H}$ I in HI 1225+01 using the VLA with 40" resolution. They deduce that the SW component is a thin rotating disk viewed nearly edge on, with an axial ratio of about 4:1. This is based upon the shape of the contours of the higher column density regions, but also on the shape of the velocity displacement as a function of position as well as the fact that such a steep inclination would explain why the peak column density is higher than in the NE component. They also point out that if the aspect ratio is $>3$ then the true (normal) surface density in the SW component is not high enough to trigger star formation (none is observed), contrary to the situation in the NE component. For this proposed geometry, $A_{\text {tot }} / A_{\text {proj }}=8$.

However, the dynamical mass for HI 1225+01 SW calculated by Chengalur et al (1995) within a radius of $3^{\prime}$ is $7 \times 10^{8} M_{\odot}$, slightly smaller than the total HI mass of HI $1225+01$ SW. An HI mass comparable to the dynamical mass is of course contrary to what is now known about the ratio of total to baryonic mass. This apparent discrepancy can be reduced in several ways. First, the HI mass refers to the total HI mass of HI 1225+01 SW while the 
dynamical mass is only calculated for the inner $3^{\prime}$. Second, the distance to HI 1225+01 may be as much as a factor of three closer than $20 \mathrm{Mpc}$, the distance assumed by Chengalur et al (Salzer et al. 1991; Impey et al. 1990). This would reduce the HI mass more than the dynamical mass. Nonetheless, the rotational velocity of HI $1225+01 \mathrm{SW}, 13 \mathrm{~km} \mathrm{~s}^{-1}$, with just a small correction for inclination if the Chengalur et al geometry is correct, is extremely low for the HI mass, even at the near distance - see the Tully-Fisher type relation between rotational speed and HI mass derived by McGaugh et al. (2000). Indeed, a reasonable interpretation of the observed morphology of HI $1225+01 \mathrm{SW}$ is that it has two spiral arms elongated along the line towards HI $1225+01$ NE, with a more face-on inclination.

Accordingly, we must conclude that the geometry is uncertain. The extremes are represented by the $4 / 1$ inclined disk, with $A_{t o t} / A_{\text {proj }}=8$, and a face-on disk, with $A_{t o t} / A_{\text {proj }}=2$. For comparison, a sphere gives $A_{t o t} / A_{\text {proj }}=4$. An infinitely long cylinder with the long axis in the plane of the sky, gives $A_{t o t} / A_{\text {proj }}=\pi$; this factor is larger if the cylinder is inclined to the line of sight. An ellipsoid rotated about its major axis and with a major to minor axis ratio of 4 (perhaps a reasonable model for a tidal feature) gives $A_{\text {tot }} / A_{\text {proj }} \approx 3.22$. Again, this factor would be slightly larger if the major axis is inclined somewhat to the line of sight. In the discussion in $\S 4 \mathrm{We}$ adopt $A_{t o t} / A_{\text {proj }}=4$ as a fiducial factor, recognizing that there is an uncertainty of about two in either direction. We will refer to the $A_{\text {tot }} / A_{\text {proj }}$ values of 2, 4, and 8 as "low", "fiducial", and "high" in the following discussion. 


\section{RESULTS AND DISCUSSION}

\subsection{Inferred limits on the Radiation Field and Comparison with Previous Results}

Using the values for the geometrical factors just discussed, the $8 \mathrm{mR}$ upper limit for the $\mathrm{H} \alpha$ flux, and values of $f_{a}$ and $f_{H \alpha}$ from equation (8) we obtain values for the one sided ionizing flux of $\Phi=10.1,5.04$, and $2.52 \times 10^{3}$ photons $\mathrm{cm}^{-2} \mathrm{~s}^{-1}$ for the low, fiducial and high values of the geometrical correction factor $A_{t o t} / A_{\text {proj }}$. The first two of these are within the range inferred by Maloney (1993), while the third is about a factor of two lower, though it should be remembered that our surface brightness value is an upper limit. Expressing the radiation field as $J_{\nu}=J_{\nu_{0}}\left(\nu / \nu_{0}\right)^{-\alpha}$, we obtain $J_{\nu_{0}}=\alpha \pi^{-1} h \Phi$, where $h$ is Planck's constant and $\alpha$ is the spectral index. Assuming the SRGPF value for the spectral index of $\alpha=1.8$, the corresponding upper limits for the mean intensity at the Lyman limit are $J_{\nu_{0}}=0.96,1.92$, and $3.84 \times 10^{-23} \mathrm{ergs} \mathrm{cm}^{-2} \mathrm{~s}^{-1} \mathrm{sr}^{-1}$. The local unshielded metagalactic

photoionization rate is calculated from this as $\Gamma=4 \pi J_{\nu_{0}} \alpha_{\nu_{0}} h^{-1}(\alpha+3)^{-1}$, which yields $\log (\Gamma)=-13.62,-13.32$, and -13.02 , where $\Gamma$ has the dimensions $\mathrm{s}^{-1}$. Had we adopted a different spectral index (e.g., 1.0) the corresponding numbers for our fiducial value of $A_{\text {tot }} / A_{\text {proj }}=4$ of $\Phi, J_{\nu_{0}}$ and $\log (\Gamma)$ would be $5.92 \times 10^{3}, 1.24 \times 10^{-23}$ and -13.43 , respectively. If our possible detection at $4 \mathrm{mR}$ should ultimately be confirmed, the actual values would of course all be lower by a factor of two.

A review of recent attempts to detect, or set limits on, the intensity of the ionizing background may be found in SRGPF. Some of these are summarized in their Table 1. It should be noted however that in the paper by Donahue, Aldering \& Stocke (1995), their definition of $\Phi$ is in terms of the ionizing specific intensity, not the flux. They also assume a value for $f_{a}$ of 1.0 , so their numbers in their Table 2 cannot be compared directly with ours. Their upper limit on the H $\alpha$ surface brightness from HI $1225+01 \mathrm{SW}$ is about $65 \mathrm{mR}$. 
The H $\alpha$ surface brightness limit of VWRH $(20 \mathrm{mR})$ is the lowest surface brightness limit we are aware of prior to the current work, which is about 2.5 times higher than our new upper limit. Our use of different assumptions about the values of $f_{a}$ and $f_{H \alpha}$ and the range of the projection factors considered here (VWRH assumed a face-on slab) results in our current value for $\Phi$ which ranges between about 2.5 to 10 times lower than that quoted in VWRH.

\subsection{Comparison with Estimates of AGN and Hot Star Contributions to the Background}

A recent careful analysis of the contributions expected from both AGNs and hot stars to the ionizing background has also been carried out in SRGPF as well as estimation of the uncertainties in these estimates. In principle, these contributions are directly observable independent of cosmological parameters, but of course local H I prevents radiation near the Lyman limit from penetrating into the disk of the galaxy along essentially all lines of sight from the sun. In practice, according to SRGPF, the two largest uncertainties in the estimate of the contributions from the AGNs are the UV luminosity functions for the AGNs and the assessment of the amount of attenuation from the cumulative absorption of intervening $\mathrm{H} \mathrm{I}$ between ourselves and the AGNs.

As noted above, SRGPF derive a best estimate for the UV spectral index of 1.8 which we have adopted. For the value of the specific intensity at the Lyman limit they quote

$$
J_{\nu_{0}, a g n}=1.3_{-0.5}^{+0.8} \times 10^{-23} \mathrm{ergs} \mathrm{cm}^{-2} \mathrm{~s}^{-1} \mathrm{~Hz}^{-1} \mathrm{sr}^{-1}
$$

For the contributions from hot young stars embedded in galaxies the luminosity function of ionizing radiation is also somewhat uncertain, but the dominant uncertainty in this case is the fraction $f_{\text {esc }}$ of ionizing photons which escape the galaxy. SRGPF thus characterize this 
fraction as a free parameter but favor a value of order $5 \%$, and for this contribution quote

$$
J_{\nu_{0}, \text { stars }}=1.1_{-0.7}^{+1.4} \times 10^{-23} \text { ergs } \mathrm{cm}^{-2} \mathrm{~s}^{-1} \mathrm{~Hz}^{-1} \mathrm{sr}^{-1}
$$

the sum of which is to be compared to our $2 \sigma$ upper limits of $J_{\nu_{0}}$ which range from 0.96 to $3.84 \times 10^{-23} \mathrm{ergs} \mathrm{cm}^{-2} \mathrm{~s}^{-1} \mathrm{~Hz}^{-1} \mathrm{sr}^{-1}$.

If we examine the various combinations of the "best", "upper" and "lower" $1 \sigma$ estimates in SRGPF we find that our largest value (for the face-on projection) is compatible for all but the largest of these combinations. For our fiducial value of the projection factor, our limit is compatible with, but slightly lower than, the sum of the two "best" contributions.

\subsection{Relevance to the $\operatorname{Ly} \alpha$ Simulations}

The range of our upper limits on the inferred $\mathrm{z} \sim 0$ photoionization rate of $\log \Gamma=$ -13.6 to -13.0 may be compared with that inferred from very recent numerical simulations of the Ly $\alpha$ forest by Davé \& Tripp (2001).

The recent advances in both computing power and numerical simulation techniques have produced results for the uncondensed gas giving rise to the Ly $\alpha$ forest which reproduce with impressive faithfulness the observed properties of the Ly $\alpha$ absorption lines over the entire range of observed redshifts from $\sim 0$ to $\sim 5$. Assuming a specific cosmological model, it is possible to infer, at any epoch, the value of the ionizing flux which can be compared with empirical determinations such as the one we have presented above.

The basic argument is as follows: A complete specification of the cosmological param-

eters (e.g., $H_{0}, \Omega_{\text {bary }}, \Omega_{\Lambda}$, the $\mathrm{H} / \mathrm{He}$ ratio, and, e.g. an LCDM initial perturbation model) completely specifies the total baryon density at any redshift, and, in principle the course of 
the entire process of gas condensation. The simulations thus yield estimates of the actual density of hydrogen nuclei at any point in the spatial grid and at any redshift, along with the velocity field of that material, and thus the local column density of hydrogen nuclei. Comparing these simulations with the observed values of the frequency of the $\mathrm{H}$ I absorption lines one can then infer the required fraction of hydrogen nuclei which are neutral and thus the H I photoionization rate, $\Gamma$. Davé \& Tripp (2001) deduce a value of $\log \Gamma=-13.3 \pm 0.7$ at a mean redshift of 0.17 , and a rough extrapolation of this estimate to zero redshift yields a value of about -13.5 , which is compatible with our range of upper limits. Of course there are considerable uncertainties in the simulations themselves, in addition to the basic cosmological parameters, but these are most severe for the highest density regions where the relatively rare higher column density H I systems are formed. With further improvements in the sensitivity of the $\mathrm{H} \alpha$ measurements, improved data on the density of the low redshift Ly $\alpha$ forest line density, and refinements in the simulations themselves, it will be possible to impose a constraint on the combination of the cosmological parameters which are involved in the Davé \& Tripp (2001) estimate, especially those that yield the present epoch baryon density.

\subsection{Caveats}

As our detailed discussion of the reduction procedures and our final spectra make clear, there are certainly uncertainties in our fitted value for the $\mathrm{H} \alpha$ surface brightness and its upper limit. However, the uncertainty in the geometry of HI $1225+01 \mathrm{SW}$ also introduces an additional major uncertainty.

There is also the possibility that our (implicit) assumption that the H I gas has a pro-

jected filling factor near unity is wrong. Suppose in fact the H I gas in the cloud has already undergone significant fragmentation on scales much smaller than the $21 \mathrm{~cm}$ resolution, with 
each "cloudlet" having H I column densities substantially higher than that inferred under the assumption that no such small scale structure exists. This implies a corresponding reduction in the actual solid angle subtended by the high column density fragmented gas. In such circumstances the surface brightness of $\mathrm{H} \alpha$ from each little cloudlet would be only slightly increased from that expected in the absence of fragmentation, however the total $\mathrm{H} \alpha$ flux would be reduced by the projected area filling factor, and the true value of the photoionization value would be higher than that we have inferred. We consider this scenario rather unlikely since one would expect star formation to be underway in at least some portions of the structure if such gas fragmentation had already occurred to such an extent. It is also possible that the tidal interaction between the NE and SW components has produced filamentary or clumpy HI structure as sometimes seen in higher linear resolution observations of tidally interacting galaxies.

We have also considered the possibility of significant amounts of internal extinction of $\mathrm{H} \alpha$ in the SW component by dust. Even for a normal dust/gas ratio, for $N(H I)=10^{19}$ $\mathrm{cm}^{-2}$ the extinction is negligible; however, for the peak observed column density of $1.1 \times 10^{21}$ $\mathrm{cm}^{-2}$ in the $40^{\prime \prime}$ resolution map of Chengalur, Giovanelli \& Haynes (1995), the emission in that direction could be attenuated to $75 \%$ of the extinction-free value. However, most of the $6590.4 \AA$ ring lies toward regions of the target where the column density is much lower (see Figure 3), with a median column density of $4 \times 10^{19} \mathrm{~cm}^{-2}$. In any case, a normal dust/gas ratio in a cloud in which no star formation seems to have occurred seems unlikely.

It would be useful to reobserve the SW component of HI 1225+01 with improved sensitivity in order to see if $\mathrm{H} \alpha$ at the level of $\sim 4 \mathrm{mR}$ is really present. However, the ambiguity in the geometry of HI $1225+01$ SW remains a difficuly. It would be very interesting for numerical simulations of interacting galaxies to be run to see if the morphology and velocity fields of the entire HI 1225+01 complex can be understood, and this may shed some light on 
the correct geometry. Beyond the issue of the geometry however, investigation of the initial conditions and subsequent evolution of the rare large isolated H I clouds such as HI 1225+01 in which little or no star formation has taken place would seem a worthwhile program. In any event, it will be important to observe with increased sensitivity several other suitable targets, since although the SW HI 1225+01 component has some features which make it an attractive target, the [N II] Galactic emission and the uncertain geometry make a definitive determination of the background flux problematic.

The expert assistance of Bill Kunkel and Oscar Duhalde in resolving technical problems at the telescope is gratefully acknowledged. RJW thanks Romeel Davé for helpful discussions on the Ly $\alpha$ forest simulations and Don Osterbrock and Tom Slanger for enlightening discussions and data concerning the night sky spectrum. SNV and SV acknowledge support from NSF AST-9529167. Finally, we acknowledge the helpful comments of the referee, P.

Maloney, in particular for pointing out the dynamical objection to the Chengalur, Giovanelli \& Haynes (1995) geometrical model.

\section{REFERENCES}

Bajtlik, S., Duncan, R. C., \& Ostriker, J. P. 1988, ApJ, 327, 570

Barnes, D. G. et al. 2001, MNRAS, 322, 486

Bechtold, J. 1994, ApJS, 91, 1

Beers, T. C., Flynn, K., \& Gebhardt, K. 1990, AJ, 100, 32

Bertin, E. \& Arnouts, S. 1996, A\&A, 117, 393

Bland-Hawthorn, J., Freeman, K. C., \& Quinn, P. J. 1997, ApJ, 490, 143 
Bland-Hawthorn, J. \& Putnam, M. 2001, in ASP Conf. Series 000, Gas and Galaxy Evolution, ed. J.E. Hibbard, M.P. Rupen, \& J.H. van Gorkom (San Francisco:ASP), 000

Burton, W. B. \& Hartmann, D. 1994, Ap\&SS, 217, 189

Carignan, C., \& Purton, C. 1998, ApJ, 506, 125

Chengalur, J.N., Giovanelli, R., \& Haynes, M. 1995, AJ, 109, 2415

Davé, R. \& Tripp, T. M. 2001, ApJ, 553, 000 (in press; astro-ph/0101419)

Donahue, M., Aldering, G., \& Stocke, J. T. 1995, ApJ, 450, L45

Dove, J. B. \& Shull, J. M. 1994, ApJ, 423, 196

Felten, J. E. \& Bergeron, J. 1969, ApL, 4, 155

Giovanelli, R. \& Haynes, M. P. 1989, ApJ, 346, L5

Giovanelli, R., Williams, J. P., \& Haynes, M. P. 1991, AJ, 101, 1242

Impey, C., Bothun, G., Malin, D., \& Staveley-Smith, L. 1990, ApJ, 351, L33

Maloney, P. 1993, ApJ, 414, 41

McGaugh, S., Schombert, J., Bothun, G., \& de Blok, W. 2000, ApJ, 533, L99

Osterbrock, D. E. 1989, Astrophysics of Gaseous Nebulae and Active Galactic Nuclei (Mill Valley: University Science Books)

Osterbrock, D. E., Fulbright, J.P., \& Bida, T.A. PASP 109, 614.

Pengelly, R. M., \& Seaton, M. J. 1964, MNRAS, 127, 145

Rand, R. J., Kulkarni, S. R., \& Hester, J. J. 1990, ApJ, 352, L1 
Salzer, J. J., di Serego Alighieri, S., Matteucci, F., Giovanelli, R., \& Haynes, M. P. 1991, AJ, 101, 1258

Schlegel, D. J., Finkbeiner, D. P., \& Davis, M. 1998, ApJ, 500, 525

Scott, J., Bechtold, J., Dobrzycki, A., \& Kulkarni, V. P. 2000, ApJS, 130, 67

Shull, J. M., Roberts, D., Giroux, M. L., Penton, S. V., \& Fardal, M. A. 1999, AJ, 118, 1450 $(\mathrm{SRGPF})$

Slanger, T. G. , Cosby, P. C. , Huestis, D. L. , \& Osterbrock. D. E. 2000, J. Geophys. Res., 105, 20557

Stocke, J. T., Case, J., Donahue, M., Shull, J. M., \& Snow, T. P. 1991, ApJ, 374, 72

Sunyaev, R. A. 1969, ApL, 3, 33

Tufte, S. L., Reynolds, R. J., \& Haffner, L. M. 1998, ApJ, 504, 773

Turner, N. J. J. \& MacFadyen, A. 1997, MNRAS, 285, 125

Veilleux, S., Cecil, G., \& Bland-Hawthorn, J. 1995, ApJ, 445, 152

Vogel, S. N., Weymann, R., Rauch, M., \& Hamilton, T. 1995, ApJ, 441, 162 (VWRH)

Wakker, B. P. 1990, Ph.D. Thesis, University of Groningen

Wakker, B. P. \& van Woerden, H. 1997, ARA\&A, 35, 217

Walsh, T., Staveley-Smith, L., \& Oosterloo, T. 1997, AJ, 113, 1591

Weiner, B. J. 2001, in preparation

Weiner, B. J., Vogel, S. N., \& Williams, T. B. 2001, in ASP Conf. Series 000, Gas and Galaxy Evolution, ed. J.E. Hibbard, M.P. Rupen, \& J.H. van Gorkom (San Francisco:ASP), 000 (astro-ph/0008263) 
Weiner, B. J. \& Williams, T. B. 1996, AJ, 111, 1156

This preprint was prepared with the AAS LATEX macros v5.0. 
This figure "fig1.gif" is available in "gif" format from: http://arxiv.org/ps/astro-ph/0107534v1 
This figure "fig3.gif" is available in "gif" format from: http://arxiv.org/ps/astro-ph/0107534v1 